\title{
Two-Step Phase Retrieval Algorithm Using Single-Intensity Measurement
}

\author{
Cheng Zhang $\mathbb{D},{ }^{1,2}$ Meiqin Wang $\mathbb{D}^{1},{ }^{1}$ Qianwen Chen, ${ }^{1}$ Dong Wang, ${ }^{1}$ and Sui Wei ${ }^{1}$ \\ ${ }^{1}$ Key Laboratory of Intelligent Computing and Signal Processing, Ministry of Education, Anhui University, Hefei, \\ Anhui Province 230039, China \\ ${ }^{2}$ Key Laboratory of Modern Imaging and Displaying Technology, Anhui University, Hefei, Anhui Province 230039, China
}

Correspondence should be addressed to Meiqin Wang; 2417724433@qq.com

Received 13 July 2018; Accepted 30 August 2018; Published 1 October 2018

Academic Editor: Xiaowei Li

Copyright (C) 2018 Cheng Zhang et al. This is an open access article distributed under the Creative Commons Attribution License, which permits unrestricted use, distribution, and reproduction in any medium, provided the original work is properly cited.

\begin{abstract}
Aiming at the problem that the single-intensity phase retrieval method has poor reconstruction quality and low probability of successful recovery, an improved method is proposed in this paper. Our method divides the phase retrieval into two steps: firstly, the GS algorithm is used to recover the amplitude in the spatial domain from the single-spread Fourier spectrum, and then the classical GS algorithm using two intensity measurements (one is recorded and the other is estimated from the first step) measurements is used to recover the phase. Finally, the effectiveness of the proposed method is verified by numerical experiments.
\end{abstract}

\section{Introduction}

Most of the information in the optical field is contained in the phase, such as the depth and shape of the object and so on. The oscillation frequency of the optical field is as high as $10^{15} \mathrm{~Hz}$. However, existing detection devices can only record intensity, directly [1]. Phase retrieval method recovers lost phase from recorded intensity measurements with some prior knowledge, which plays an important role in the imaging field. Phase retrieval problem arises in numerous of areas, such as crystallography [2-4], optics [5-9], astronomical imaging [10], microscopy [11, 12], biomedical [13], and holographic imaging[14, 15].

In 1972, Gerchberg and Saxton first proposed an alternate projection-based phase retrieval algorithm, the GerchbergSaxton (GS) algorithm [16]. The main idea of GS algorithm is to use the intensity of the spatial domain and the Fourier domain to recover the phase of the optical field. Subsequently, Fienup proved that the GS algorithm had obvious errordecreasing properties, Error Reduction (ER) algorithm, and Hybrid Input-Output (HIO) algorithm were proposed [17]. At present, ER algorithm and HIO algorithm are considered to be the most effective methods in the field of phase retrieval [18]. Since the above algorithms are only suitable for a positive linear transformation system, nobody cares about any linear transformation system. Therefore, Yang and $\mathrm{Gu}$ proposed the amplitude-phase detection theory in arbitrary linear transformation systems, namely, the Yang-Gu (Y-G) algorithm [19]. In 2015, Guo et al. optimized the iterative algorithm and proposed two improved GS iterative phase retrieval algorithms, which were the spatial phase perturbation GS algorithm and the combined GS HIO algorithm. For the two improved algorithms, the squared value of the squared error value rapidly drops to an acceptable value, and the lost phase can be successfully recovered in the spatial domain and Fourier domain, which means that both algorithms can jump out of the local minimum value and convergence to the global minimum [9].

The GS algorithm was originally proposed in connection with the problem of reconstructing phase given only twointensity measurements in the spatial domain and Fourier domain, i.e., given two intensity measurements (one is recorded in the spatial domain and the other is estimated in the Fourier domain) measurements. Unfortunately, such two intensity measurements at two different planes cannot be measured in some cases. Therefore, a GS algorithm using single-intensity measurement with some prior knowledge combined was proposed to recover the phase. However the 


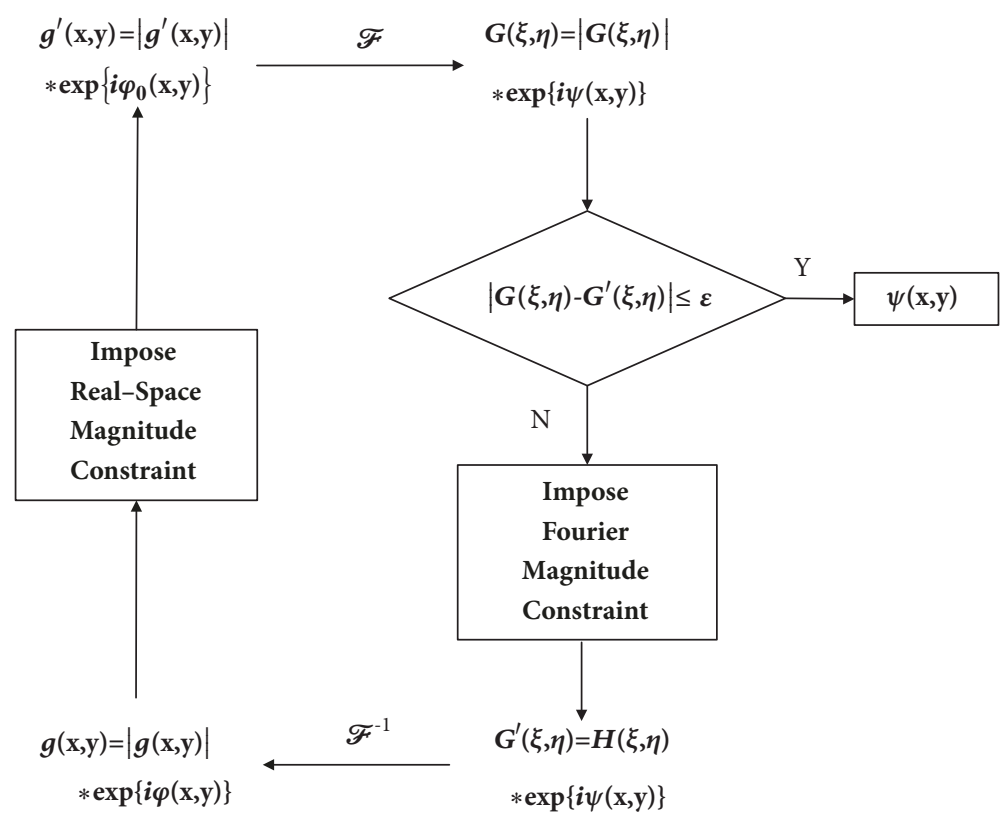

FIGURE 1: Flow chart of classical GS algorithms using two intensity measurements, i.e., TIPR.

single-intensity phase retrieval method has the drawbacks of poor reconstruction quality and low probability of successful recovery. This paper proposes an improved method based on single-intensity measurement, Two-Step Phase Retrieval (TSPR) from single-intensity measurement algorithm. The main idea of the TSPR algorithm is to first recover the amplitude (i.e., the square root of the intensity) in the spatial domain using the Single-Intensity Phase Retrieval (SIPR) algorithm with some prior knowledge and then recover the phase in the spatial domain using the Two-Intensity Phase Retrieval (TIPR) algorithm. Experimental results demonstrate that our proposed TSPR algorithm can effectively enhance the quality of phase recovered and the probability of successful recovery.

\section{Basic Principles}

The complex amplitude $g(x, y)$ is

$$
g(x, y)=|g(x, y)| \exp (i \varphi(x, y))
$$

Its Fourier transform form is

$$
G(\xi, \eta)=|G(\xi, \eta)| \exp (i \psi(\xi, \eta))
$$

where $g(x, y)$ and $G(\xi, \eta)$ denote the complex amplitude in the spatial domain and Fourier domain, respectively, $|g(x, y)|$ and $|G(\xi, \eta)|$ denote the amplitude in the spatial domain and Fourier domain, respectively, $\varphi(x, y)$ and $\psi(\xi, \eta)$ denote the phase in the spatial domain and Fourier domain, respectively. Phase retrieval refers to recovering the losing phase $\varphi(x, y)$ in the spatial domain from two amplitudes $|g(x, y)|$ and $|G(\xi, \eta)|$.

The flow chart of the classical GS algorithm is shown in Figure 1. This algorithm was first proposed and was easily used to solve the phase retrieval problem using two intensity measurements. $\exp \left(\mathrm{i} \phi_{0}(\mathrm{x}, \mathrm{y})\right)$ denotes the initialized phase distribution function. The algorithm repeatedly iterates between the spatial domain and the Fourier domain until the error $\varepsilon$ satisfies the termination condition [20].

\section{Two-Step Phase Retrieval Using Single- Intensity Measurement}

The TIPR algorithm uses the two intensity measurements recorded in the spatial domain and the Fourier domain, respectively, to recover the amplitude and phase in the spatial domain with high quality. Although the TIPR algorithm can achieve good results, in some case, it is difficult to obtain two intensity measurements simultaneously in the both spatial and Fourier domains.

Therefore, the SIPR algorithm is proposed, and it can recover the phase from single-intensity measurement in the Fourier domain with some prior knowledge. However, the SIPR method has low probability of successful recovery and poor quality. As shown in Figure 2, the purpose of the experiment is to test the SIPR method's performance to recover the amplitude and phase. The coded aperture is generated by a uniform distribution. The sampling rate of the coded aperture is used as a variable parameter in the experiment. The sampling rate gradually increased from 0.1 to 0.7 ; the step length is 0.05 . The experiment runs 500 times independently to compute the probability of successful recovery. If the SNR of the phase reconstructed is greater than $25 \mathrm{~dB}$, the reconstruction is deemed as successful. The probability of successful recovery versus sampling rate for both amplitude and phase is shown in Figure 2.

As depicted in Figure 2, it can be found that the SIPR method can achieve high probability of successful recovery 


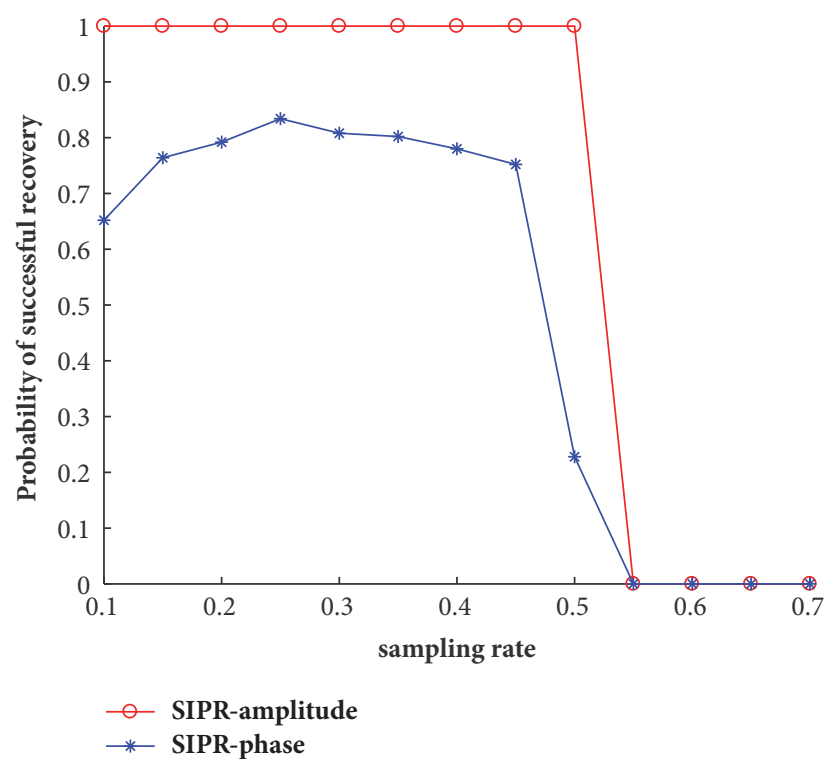

FIGURE 2: Probability of successful recovery of amplitude and phase versus sampling rate.

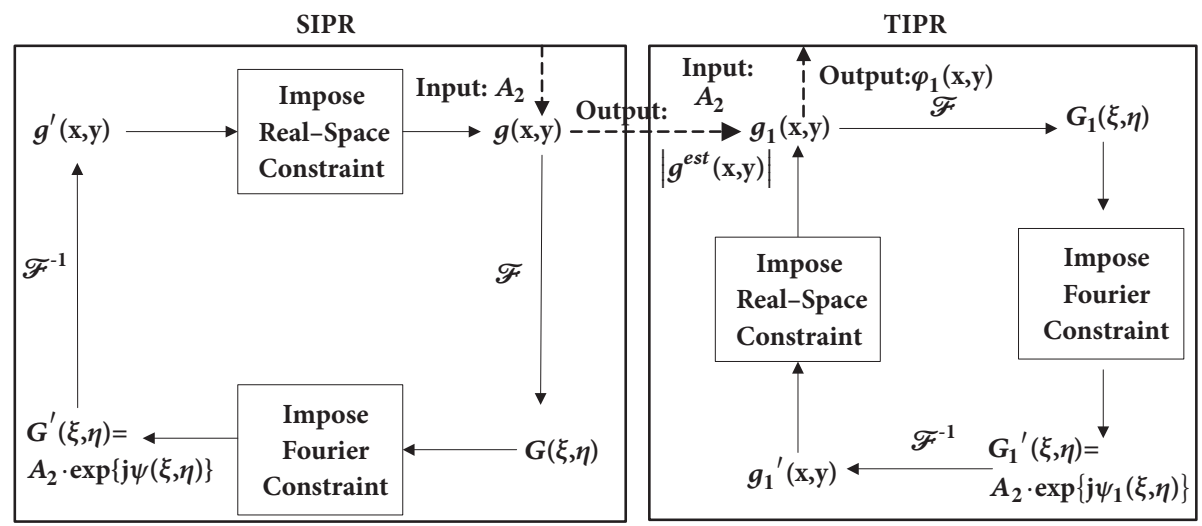

FIgURE 3: Flow diagram of the TSPR algorithm.

of the amplitude, but the probability of successful recovery of the phase is very low and unstable. Hence, our TSPR method is proposed to solve the phase retrieval problem from single-intensity measurement. Firstly, SIPR method is used to recover the amplitude in the spatial domain from the intensity in the Fourier domain and the support set in the spatial domain. Then, TIPR method using two intensity measurements (one of the two intensity measurements is recorded in the Fourier domain and the other is estimated in the spatial domain from the first step) is used to recover the phase in the spatial domain.

The flow chart of TSPR method is shown in Figure 3, which includes the following steps: (1) initialize the amplitude $A_{1}$ and phase $\varphi_{0}(\mathrm{x}, \mathrm{y})$ in the spatial domain, i.e., use allone amplitude and randomized phase. (2) Perform Fourier transform on the complex amplitudes and replace the amplitude after the Fourier transform in the Fourier domain with the recorded Fourier amplitudes $A_{2}$. (3) Perform the inverse Fourier transform on the synthesized complex amplitude in the Fourier domain and multiply it with the support in the spatial domain. (4) Repeat steps (2)-(3) until the amplitude converges in the spatial domain. (5) The classical TIPR algorithm using the recorded Fourier amplitude $A_{2}$ and the estimated amplitude $\left|g^{\text {est }}(\mathrm{x}, \mathrm{y})\right|$ from the first step is used to iteratively recover the phase in the spatial domain.

The complex amplitude of the initialization is as follows:

$$
g(x, y)=A_{1} * \exp \left[\varphi_{0}(x, y)\right]
$$

The optical setup of imaging process of the TSPR method is shown in Figure 4. The coded aperture and CCD are placed on the front and back focal planes of the lens, respectively.

Firstly, the complex optical field $u(x, y)$ is filtered through the coded aperture $M$ to obtain a complex optical field $u_{M}(x, y)$ and then imaged by a Fourier lens; finally the intensity is recorded in the CCD plane. The intensity recorded at the CCD plane is expressed as follows:

$$
I_{C C D}=\left|\mathscr{F}\left[u_{M}(x, y)\right]\right|^{2}
$$




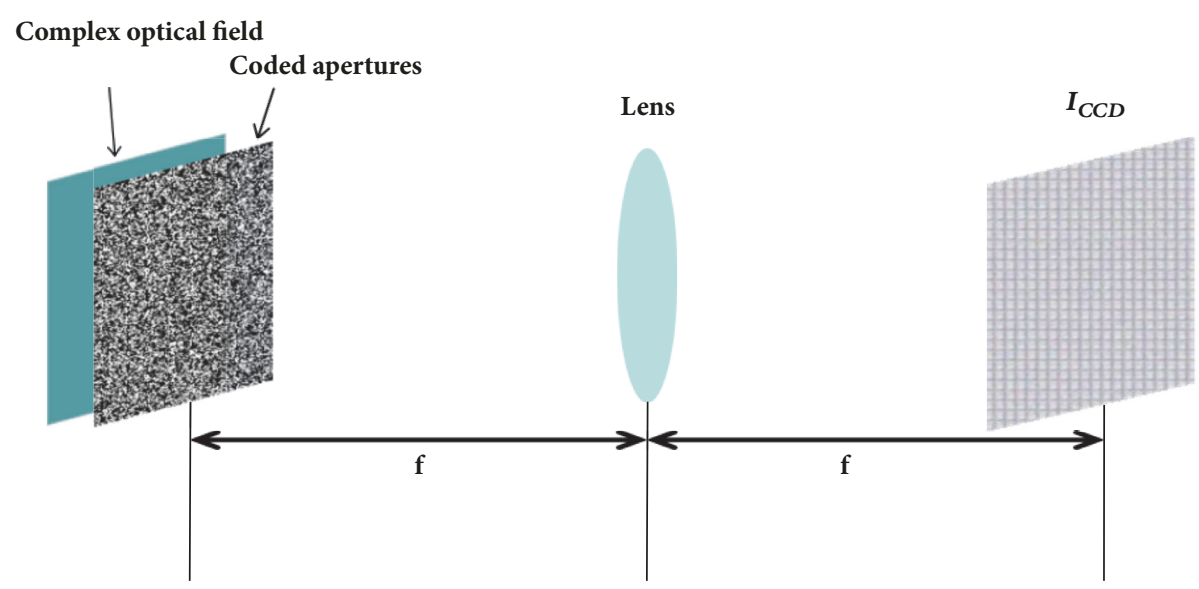

FIGURE 4: The optical setup of imaging process.

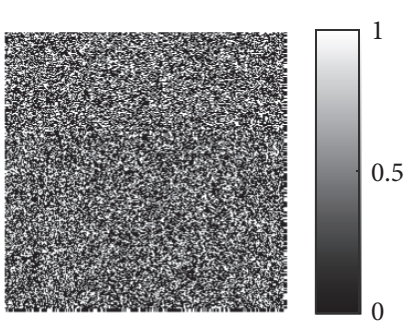

(a)

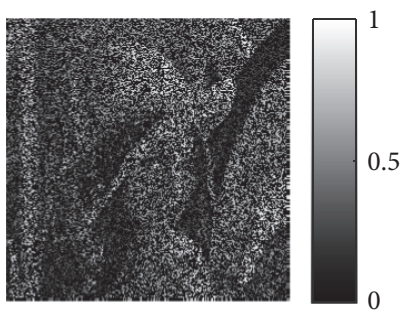

(e)

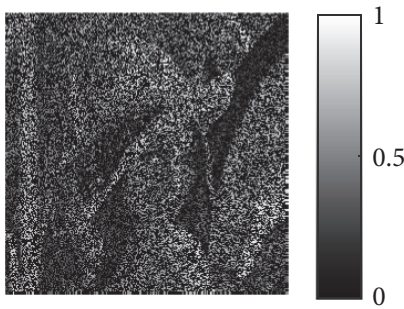

(b)

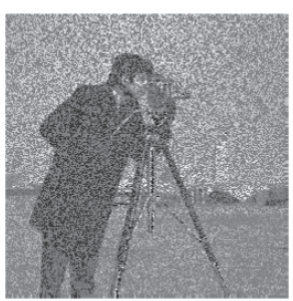

(f)

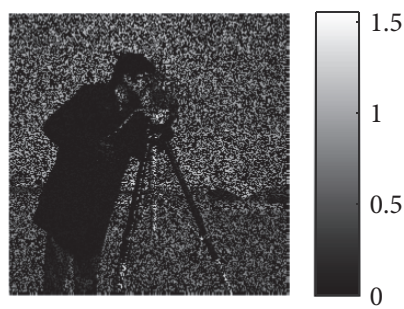

(c)
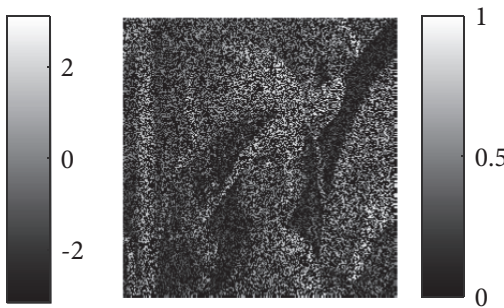

(g)

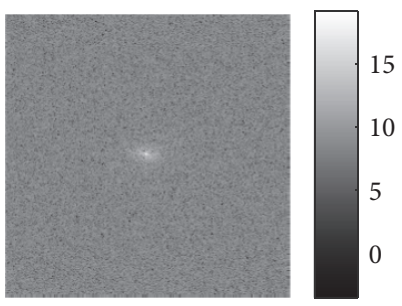

(d)

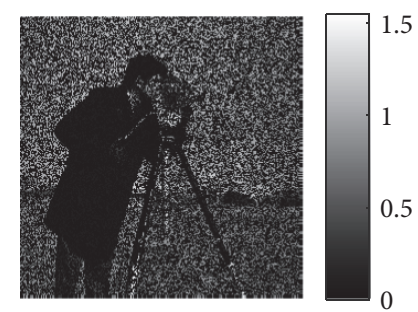

(h)

FIGURE 5: Comparison of SIPR algorithm and TSPR algorithm. (a) A 0/1 randomly distributed coded aperture. (b) and (c) are the amplitude and phase of the complex optical field behind the coded aperture. (d) The intensity recorded in the Fourier domain. (e) and (f) are the amplitude and phase recovered using SIPR algorithm. $(\mathrm{g})$ and $(\mathrm{h})$ are the amplitude and phase recovered using TSPR algorithm.

The main purpose of this paper is to recover the phase of the complex optical field $u_{M}(x, y)$ from the recorded intensity measurement $I_{C C D}$.

\section{Numerical Experiments}

In order to verify the effectiveness and superiority of our proposed method, three experiments are presented in this section. The first simulation experiment is the single reconstruction experiment. The second simulation experiment is to test the performance of TSPR algorithm under different coded apertures. The final simulation experiment is to compare the performance of the different phase retrieval algorithms.
4.1. Experiment 1: Single Reconstruction Experiment. The purpose of the experiment is to verify the feasibility of our proposed TSPR method. Two grayscale images ("Lena" and "Cameraman" with $256 \times 256$ pixels) are chosen in the experiment. The two images are multiplied by the coded aperture $(256 \times 256$ pixels $)$ to obtain the amplitude and phase, respectively. Then the complex amplitude is synthesized by amplitude and phase. The coded aperture is $0 / 1$ random distribution. The sampling rate in this experiment is 0.4 , and the phase retrieval results are shown in Figure 5.

Figure 5(a) is the coded aperture with $0 / 1$ randomly distributed, and the sampling rate is 0.4 . Figures 5(b) and 5(c) are the amplitude and phase of the complex optical field behind 


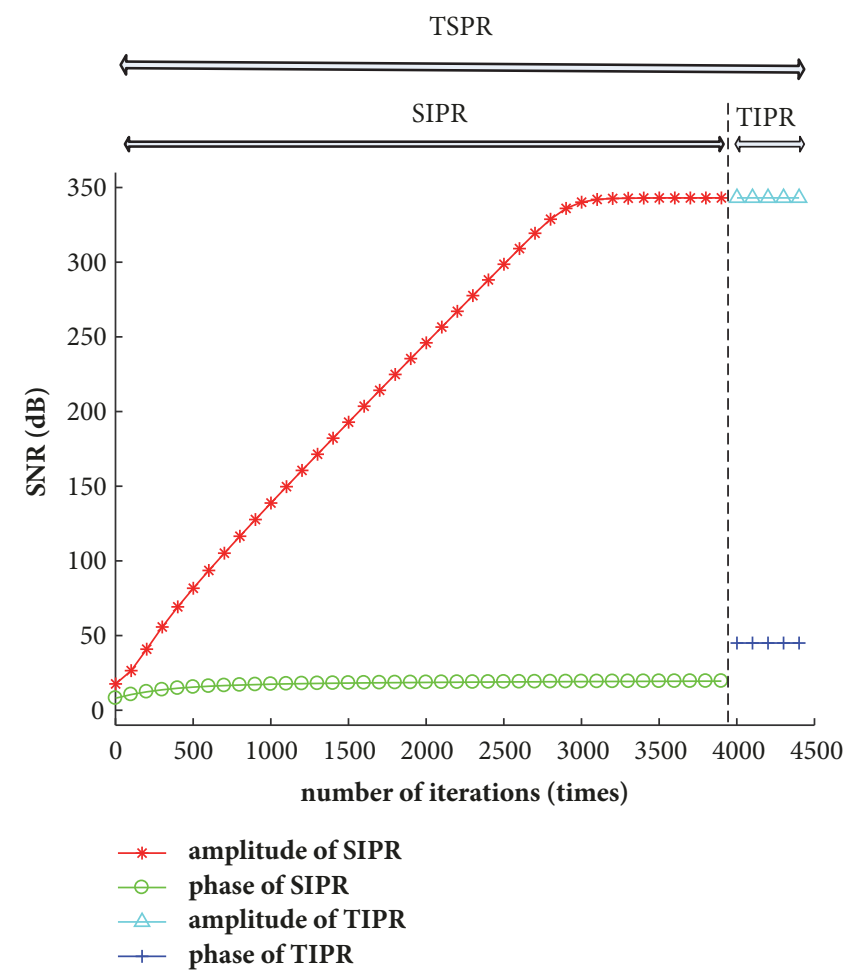

FIgURE 6: Amplitude and phase versus the number of iterations.

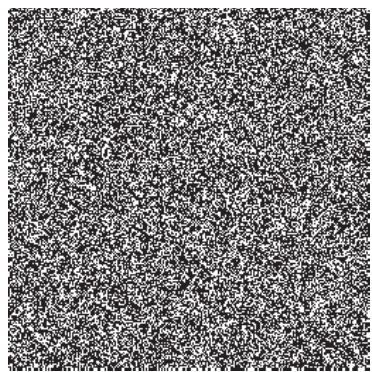

(a)

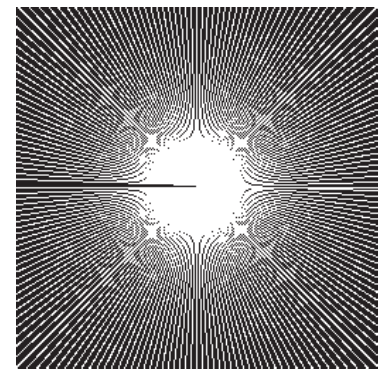

(b)

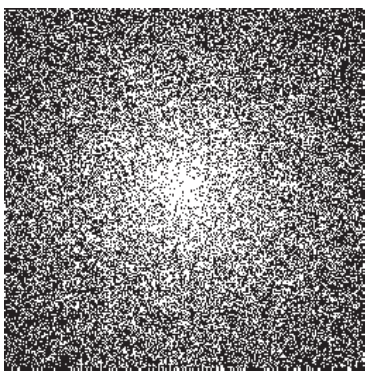

(c)

FIGURE 7: Three types of coded apertures with sampling rate of 0.3. (a) Uniform random sampling pattern. (b) Radial line sampling pattern. (c) Variable density sampling pattern.

the coded aperture to be retrieved. Figure 5(d) is the intensity recorded in the Fourier domain. Figures 5(e) and 5(f) are the amplitude and phase recovered using the SIPR algorithm, and the Signal-to-Noise Ratio (SNR) is $343.04 \mathrm{~dB}$ and 19.85 $\mathrm{dB}$, respectively. Figures $5(\mathrm{~g})$ and $5(\mathrm{~h})$ are the amplitude and phase recovered using the TSPR algorithm, and their SNR is $343.04 \mathrm{~dB}$ and $45.58 \mathrm{~dB}$, respectively. Comparing Figures $5(\mathrm{f})-5(\mathrm{~h})$, it is easy to find that the quality of phase recovered is greatly improved by our TSPR method.

The trend of the amplitude and phase with the number of iterations in our TSPR method is shown in Figure 6. In the first step (i.e., SIPR) of TSPR, the amplitude can be well recovered, but the phase recovered is poor. As the number of iterations increases, the SNR of the phase recovered can only reach about $19 \mathrm{~dB}$. However, in the second step (i.e., TIPR) of the TSPR, the SNR of the phase recovered increases as high as $45 \mathrm{~dB}$. Compared with SIPR method, TSPR method can greatly improve the SNR of phase reconstructed.

4.2. Experiment 2: Reconstruction Experiments under Different Coded Apertures. The coded aperture in Experiment 1 is a uniform random sampling pattern. The purpose of Experiment 2 is to test the performance of the TSPR method under different coded apertures.

In Figure 7, three different types of the coded apertures are selected, such as Uniform Random (UR) sampling, Radial Line (RL) sampling, and Variable Density (VD) sampling. The sampling rate of the coded aperture is used as a variable parameter in the experiment, and the sampling rate is set from 0.1 to 0.7. Each set of experiments with the 


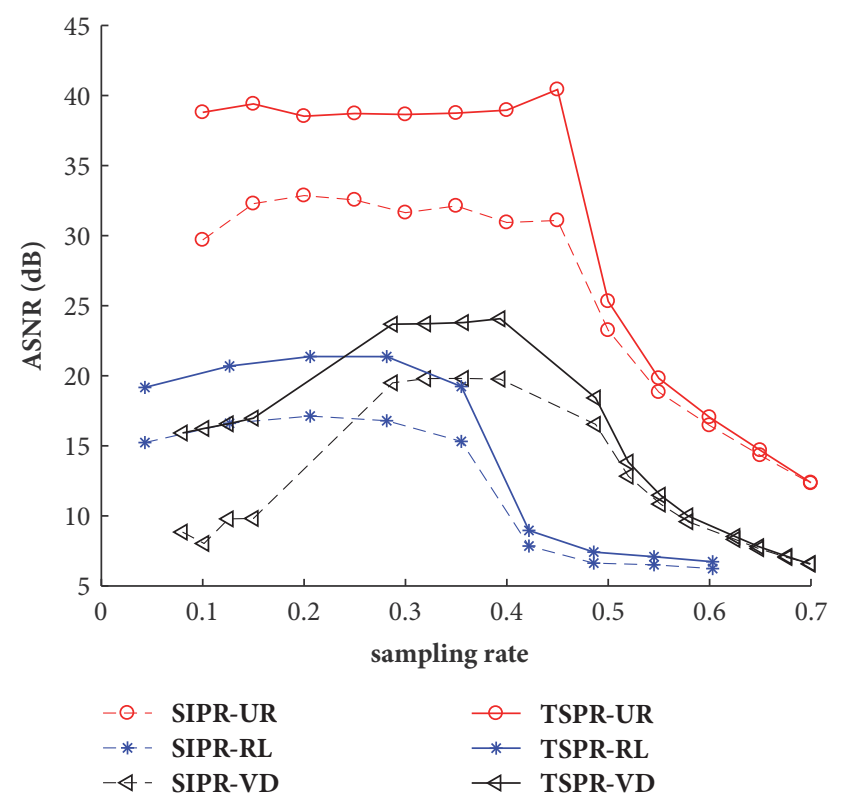

FIGURE 8: ASNR versus sampling rate.

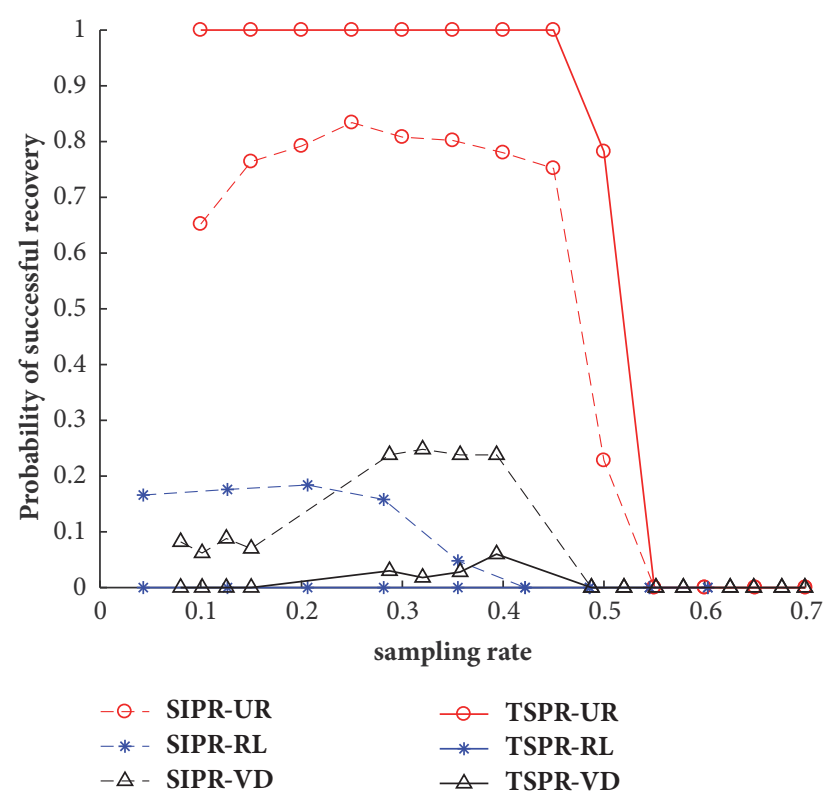

FIGURE 9: Probability of successful recovery versus sampling rate.

same parameters runs 500 times independently. Compute the recovered Average Signal-to-Noise Ratio (ASNR) and probability of successful recovery. The ASNR and probability of successful recovery versus sampling rate under different coded apertures are shown in Figures 8 and 9, respectively.

As shown in Figure 8, as the sampling rate increases, the TSPR algorithm achieves higher ASNR for phase recovered than the SIPR algorithm under three different coded apertures. In Figure 9, the best choice for coded aperture is uniform random sampling pattern, which achieve the optimal performance. The probability of successful recovery has also been significantly improved and has a good stability.

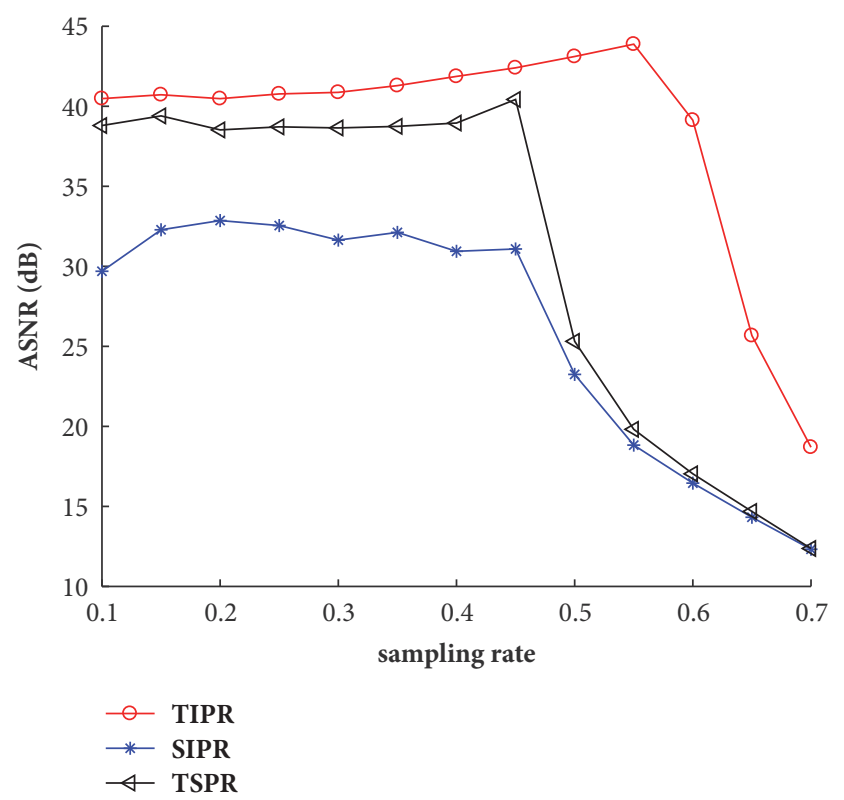

FIGURE 10: ASNR versus sampling rate.

The reason is that the support set of the uniform random sampling pattern is relatively uniform distributed in the spatial domain, while the support set of the other two coded apertures is more concentrated to the center, so the phase cannot be retrieved well.

\subsection{Experiment 3: Reconstruction Experiment under Different} Sampling Rates. The purpose of Experiment 3 is to compare the performance of phase retrieval between our TSPR method and the other two algorithms (TIPR algorithm and SIPR algorithm) under different sampling rates. The coded aperture uses a uniform random sampling pattern with a sampling rate varies from 0.1 to 0.7 and the step size is 0.05. Under the same parameters, experiments runs 500 times independently. The ASNR and probability of successful recovery versus sampling rate for different algorithms are presented in Figures 10 and 11, respectively.

As shown in Figures 10 and 11, the TIPR method is the best, the TSPR method is the second, and the SIPR method is the worst. The reason is that TIPR method has the most known information, i.e., the two intensity measurements at two different planes. The difference between the TSPR method and the SIPR method is that the TSPR method draws on the idea of the TIPR method. Firstly, use the SIPR method to estimate the amplitude in the spatial domain, then use the TIPR method to recover the phase. In summary, the TSPR method is superior to the SIPR method.

\section{Conclusion}

This paper proposes an improved TSPR algorithm to solve the problem of poor quality and low success rate of SIPR method. TSPR algorithm is completed in two steps: firstly, SIPR algorithm is used to recover the amplitude in the spatial domain, then TIPR algorithm is used to recover the phase in 


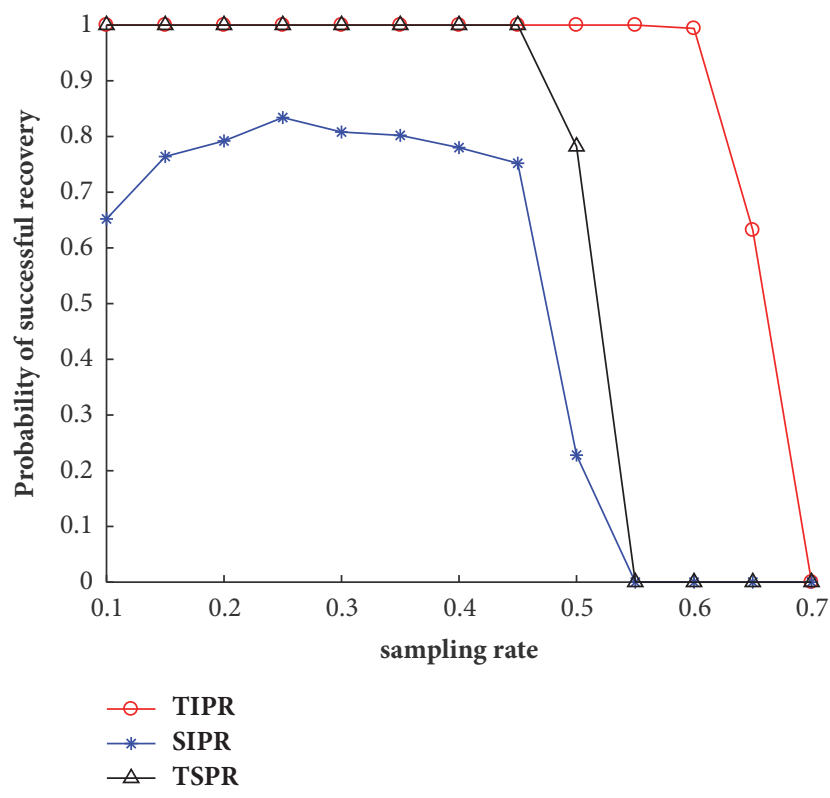

FIGURE 11: Probability of success versus sampling rate.

the spatial domain. Finally, the effectiveness and superiority of the TSPR method are verified. The experiment comparison results demonstrate that the proposed method can effectively improve the ASNR and probability of successful recovery under the same parameters. TSPR algorithm can recover the lost phase from known amplitude in the Fourier domain and support set in the spatial domain.

\section{Data Availability}

The data in this paper has been signed into a commercial agreement, so it is confidential and cannot be made public.

\section{Conflicts of Interest}

The authors declare that they have no conflicts of interest.

\section{Acknowledgments}

This work was supported by the National Natural Science Foundation of China (Grant Numbers 61501001, 61605002, 61301296, and 61377006) and the Natural Science Foundation of Anhui Province (Grant Numbers 1508085MF121, 1608085QF161).

\section{References}

[1] Y. Shechtman, Y. C. Eldar, O. Cohen, H. N. Chapman, J. Miao, and M. Segev, "Phase retrieval with application to optical imaging: a contemporary overview," IEEE Signal Processing Magazine, vol. 32, no. 3, pp. 87-109, 2015.

[2] R. W. Harrison, "Phase problem in crystallography," Journal of the Optical Society of America A: Optics, Image Science \& Vision, vol. 10, no. 5, pp. 1046-1055, 1993.
[3] R. P. Millane, "Phase retrieval in crystallography and optics," Journal of the Optical Society of America A: Optics, Image Science \& Vision, vol. 7, no. 3, pp. 394-411, 1990.

[4] J. A. Rodriguez, R. Xu, C.-C. Chen, Y. Zou, and J. Miao, "Oversampling smoothness: An effective algorithm for phase retrieval of noisy diffraction intensities," Journal of Applied Crystallography, vol. 46, no. 2, pp. 312-318, 2013.

[5] A. Walther, "The question of phase retrieval in optics," Journal of Modern Optics, vol. 10, pp. 41-49, 1963.

[6] M. C. Newton, "Compressed sensing for phase retrieval," Physical Review E: Statistical, Nonlinear, and Soft Matter Physics, vol. 85 , no. $5,2012$.

[7] J. Miao, P. Charalambous, J. Kirz, and D. Sayre, "Extending the methodology of X-ray crystallography to allow imaging of micrometre-sized non-crystalline specimens," Nature, vol. 400, no. 6742 , pp. 342-344, 1999.

[8] J. Hunt, J. Gollub, T. Driscoll et al., "Metamaterial microwave holographic imaging system," Journal of the Optical Society of America A: Optics, Image Science \& Vision, vol. 31, no. 10, pp. 2109-2119, 2014.

[9] C. Guo, S. Liu, and J. T. Sheridan, "Iterative phase retrieval algorithms. I: optimization," Applied Optics, vol. 54, no. 15, pp. 4698-4708, 2015.

[10] C. Fienup and J. Dainty, "Phase retrieval and image reconstruction for astronomy," Image Recovery: Theory and Application, vol. 231, p. 275, 1987.

[11] J. Miao, T. Ishikawa, Q. Shen, and T. Earnest, "Extending $\mathrm{X}$-ray crystallography to allow the imaging of noncrystalline materials, cells, and single protein complexes," Annual Review of Physical Chemistry, vol. 59, pp. 387-410, 2008.

[12] H. M. L. Faulkner and J. M. Rodenburg, "Movable aperture lensless transmission microscopy: A novel phase retrieval algorithm," Physical Review Letters, vol. 93, no. 2, Article ID 023903, 2004.

[13] M. Dierolf, A. Menzel, P. Thibault et al., "Ptychographic X-ray computed tomography at the nanoscale," Nature, vol. 467, no. 7314, pp. 436-439, 2010.

[14] G. Situ, J. P. Ryle, U. Gopinathan, and J. T. Sheridan, "Generalized in-line digital holographic technique based on intensity measurements at two different planes," Applied Optics, vol. 47, no. 5, pp. 711-717, 2008.

[15] D. Deng, W. Qu, W. He, Y. Wu, X. Liu, and X. Peng, "Phase retrieval for digital holographic microscopy with defocused holograms," IEEE Photonics Journal, vol. 10, no. 1, 2018.

[16] R. W. Gerchberg and W. O. Saxton, "A practical algorithm for the determination of the phase from image and diffraction plane pictures," Optik - International Journal for Light and Electron Optics, vol. 35, no. 2, pp. 237-250, 1972.

[17] J. R. Fienup, "Reconstruction of an object from the modulus of its Fourier transform," Optics Expresss, vol. 3, no. 1, pp. 27-29, 1978.

[18] J. R. Fienup, "Phase retrieval algorithms: a comparison," Applied Optics, vol. 21, no. 15, pp. 2758-2769, 1982.

[19] G. Yang, B. Gu, and B. Dong, "Theory of the amplitude-phase retrieval in any linear transform system and its applications," International Journal of Modern Physics B, vol. 07, no. 18, pp. 3153-3224, 1993.

[20] J. R. Fienup, "Phase retrieval algorithms: A personal tour," Applied Optics, vol. 52, no. 1, pp. 45-56, 2013. 

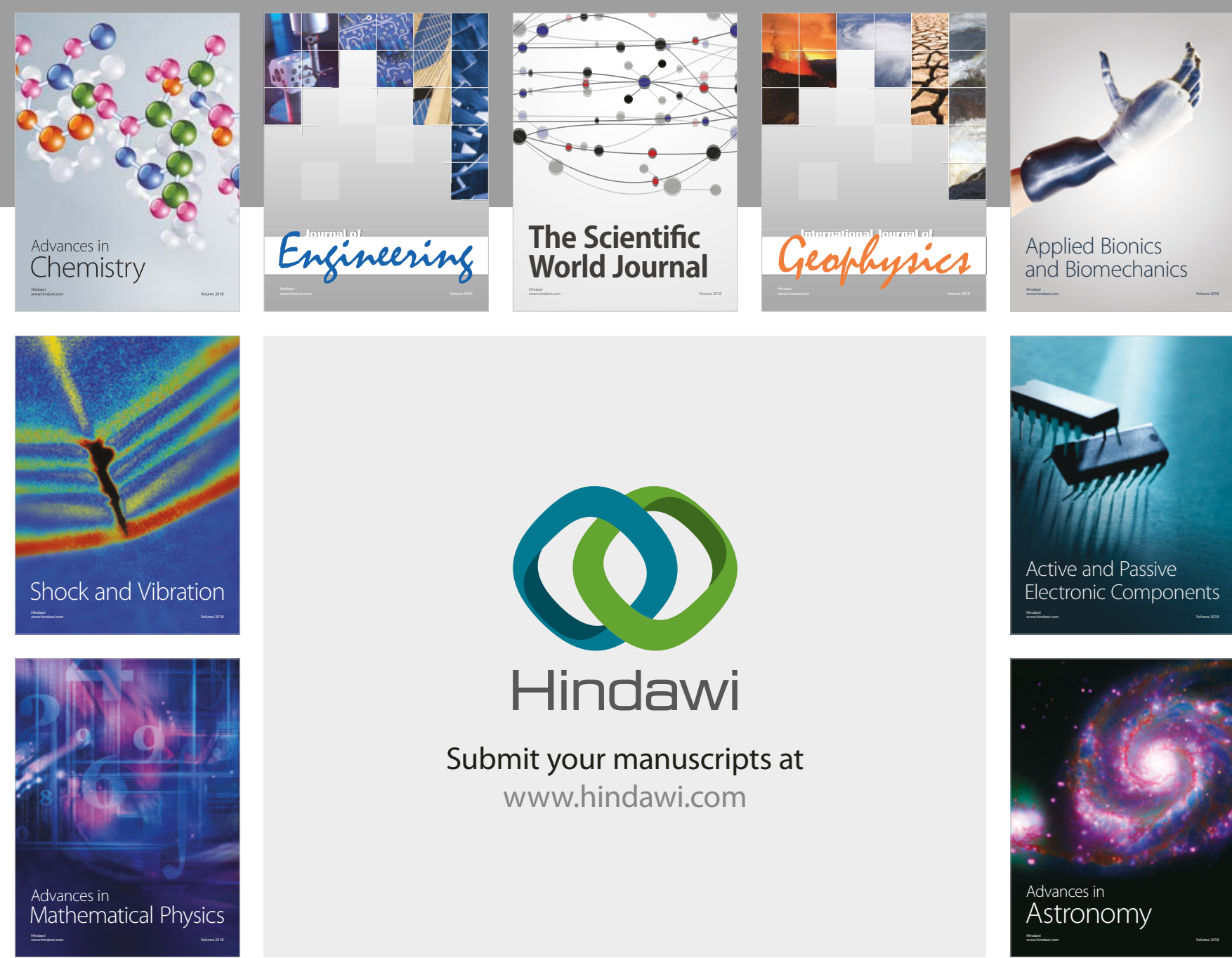

Submit your manuscripts at

www.hindawi.com

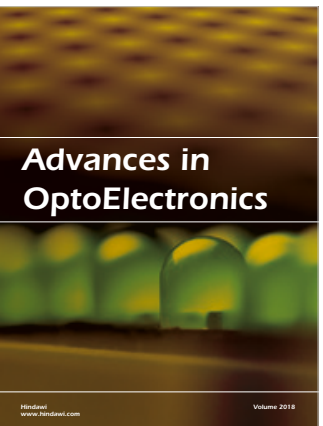

\section{Rotcting Machinery}
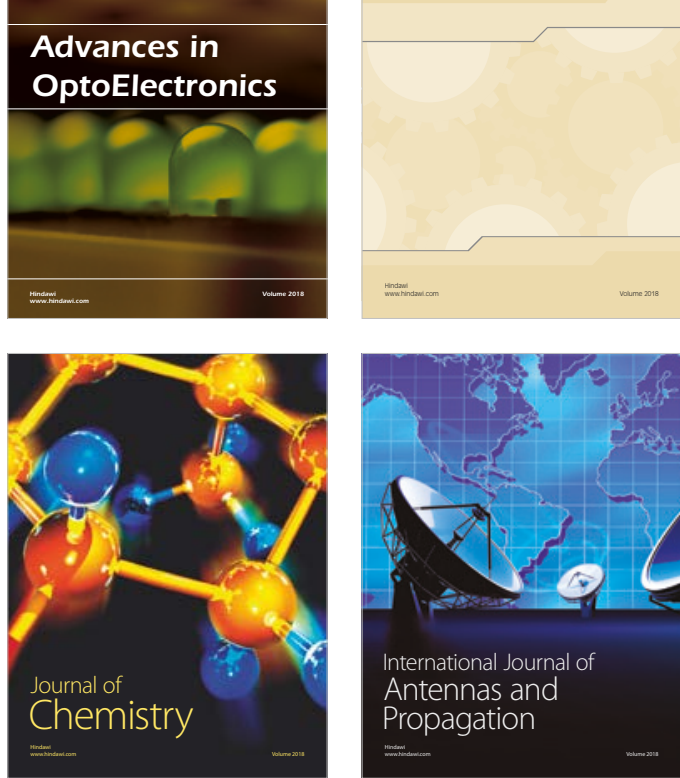

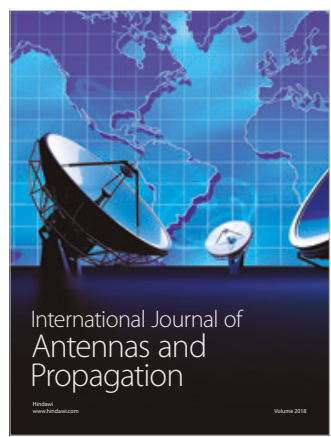

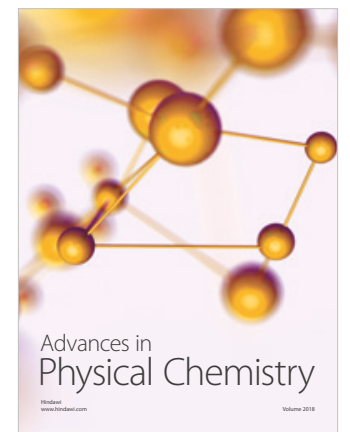

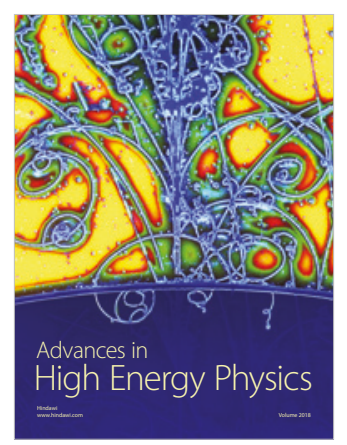

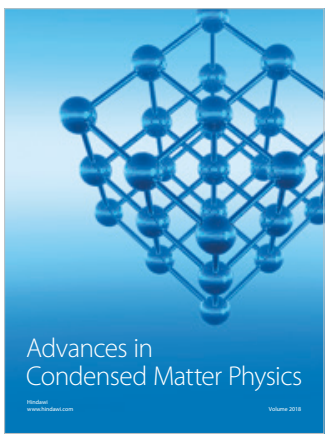

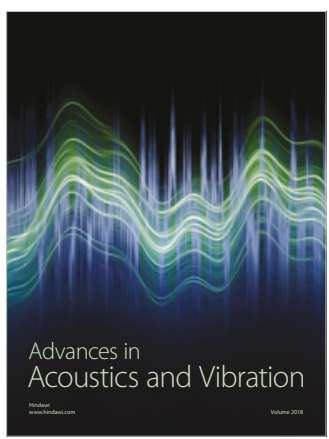

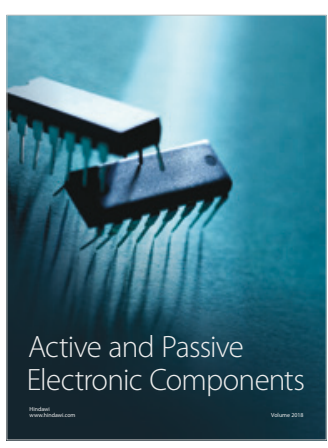
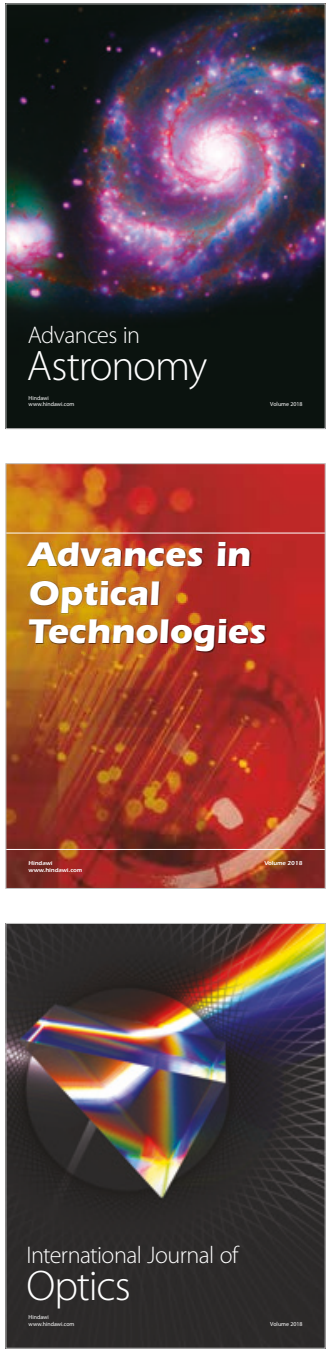